\title{
Is the PPI responsible for that inferior MI?
}

\author{
Sean R. McMahon, $M D{ }^{a}$ and W. Lane Duvall, $M D^{a}$ \\ a Division of Cardiology, Hartford Hospital, Hartford, CT
}

Received Jun 12, 2019; accepted Jun 12, 2019

doi: 10.1007/s12350-019-01795-9

\section{See related article, pp. 1611 -1619}

Gastrointestinal (GI) radiotracer uptake, including stomach, liver, and intestinal counts, in patients undergoing single photon emission computed tomography (SPECT) myocardial perfusion imaging (MPI) has the potential to degrade image quality and adversely impact interpretation. Extracardiac localization may cause increases in adjacent myocardial segments by Compton scatter or decreased counts via ramp filter artifact. The occurrences of these artifacts plague our nuclear laboratories and provide ample opportunities for improvement in imaging technique. Several factors have been identified that contribute to increased gastrointestinal uptake including supine positioning, stomach contents, timing of image acquisition, pharmacological stressors, radiotracer, and specific medications. Currently, laboratories mitigate the impact of extracardiac uptake by withholding food and oral fluids, timing injection and scanning appropriately, rescanning if inadequate images, and utilizing attenuation correction. In this issue of the Journal of Nuclear Cardiology, Singh et al propose to consider proton pump inhibitors (PPI) as a medication which increases stomach wall tracer uptake. ${ }^{1}$

GI tracer uptake, along with patient motion and soft tissue attenuation, are a daily hinderance to quality SPECT perfusion imaging. The combination of these imaging artifacts is likely responsible for the majority of preventable imaging errors and helps contribute to the less than perfect accuracy of perfusion imaging. The assessment of these artifacts is a foundational

Reprint requests: W. Lane Duvall, MD, Division of Cardiology, Hartford Hospital, Hartford, CT, USA; Lane.Duvall@hhchealth.org J Nucl Cardiol 2020;27:1620-1.

$1071-3581 / \$ 34.00$

Copyright (c) 2019 American Society of Nuclear Cardiology. component of the evaluation of every perfusion study by the technologist and physician, and in the case of GI tracer uptake and motion artifact, can be mitigated by repeat imaging when identified. All too often in this day of over-burdened health care staff, these basics of perfusion imaging techniques can be overlooked and compromises made to image quality. While any efforts to reduce the interpretation errors from imaging artifacts such as the work reported in this issue of the Journal are welcome, we must not forget that quality perfusion imaging begins and ends with the laboratory staff.

Prior work on the specific subject of stomach wall tracer uptake is quite limited and often small in regards to the sample size. The only large study on the subject by Gholamrezanezhad et al in 2006 described 1056 consecutive outpatients and found only $1.9 \%$ of patients had gastric wall hyperactivity. ${ }^{2}$ This low prevalence of stomach wall uptake is in contrast to the higher prevalence (8-36\%) seen in the current study in the Journal. Two relatively small prospective observational studies comparing stomach uptake in patients found no association with $\mathrm{H} 2$ antagonists and a strong association with prolonged PPI use and increased stomach wall activity. ${ }^{3,4}$ Goel et al described this phenomenon in a 2009 observational study including 121 patients undergoing SPECT with $99 \mathrm{mTc}$-sestamibi. Of the 26 patients on a PPI, $21(81 \%)$ had stomach wall uptake whereas only 9 of $95(9 \%)$ not on PPI were found to have stomach wall uptake $(P<0.01){ }^{3}$ Another similar study with 127 patients imaged with $99 \mathrm{mTc}$-tetrofosmin found stomach wall uptake more frequently in patients on PPI's (22\% vs $7 \%, P=0.017){ }^{4}$

In this issue, Singh et al add to the limited data on this phenomenon. One hundred and fifty-six patients were prospectively studied in a single center trial in an effort to quantify stomach uptake in patients with and without acid suppressive therapy including PPI's and H2 antagonists. This included 47 patients on a PPI, 19 patients on $\mathrm{H} 2$ antagonists, and an intervention group consisting of 42 patients on PPI therapy who were asked to discontinue treatment three days prior to MPI. The 
findings are consistent with previously reported results suggestive that PPI's but not $\mathrm{H} 2$ antagonists increase stomach wall uptake. In patients taking PPI 36\% had stomach wall uptake compared to only $8 \%$ in the control group $(P=0.002)$. This effect was seen predominately in patients undergoing vasodilator stress. Furthermore, this study adds to the literature the novel finding that patients stopping the PPI three days prior to imaging had no significant difference in stomach wall uptake compared to the control group $(9.5 \%$ vs $8 \%, P=0.84)$.

The study suggests that patients taking PPI's may be better imaged after holding PPIs for 3 days prior to imaging, especially in those who are unable to exercise. Prior to broadly adopting this practice, a randomized trial or substantially larger retrospective study should be performed to answer several remaining questions. From our current understanding it is difficult to know the impact of stomach wall uptake on lab workflow or clinical interpretation, and in fact the largest study on the subject found only $1.9 \%$ of patients were affected by this condition. This can be quantified in a larger trial assessing the number of rescans and false positive results. Image interpretation should be assessed both before and after rescanning the patient and correlated with more precise quantification of stomach wall uptake. One particular advantage of assessing these downstream effects of stomach wall uptake would be eliminating the subjectivity of differentiating stomach wall uptake from other sub-diaphragmatic activity as all of this activity, be it liver, intestine, or stomach, can adversely affect image interpretation. Furthermore, more precise quantification of the stomach wall uptake in relation to the time interval between injection and imaging, stressor type, radiotracer (sestamibi vs tetrofosmin), duration of PPI therapy, and time of PPI discontinuation would be pertinent. Further work could also assess the mechanism behind stomach wall uptake in patients taking a PPI.

Practically, implementing a strategy of screening and contacting each patient to discontinue their PPI prior to SPECT MPI is burdensome and may at times be impractical (for instance in emergency department patients or inpatients), especially when the scope of the problem of stomach wall uptake is not well understood.
Several days to a week prior to their study patients would need to be assessed for PPI use and indication, recognizing that some patients should not be taken off of acid suppressive therapy for any significant length of time. Patients with active ulcerative disease or recent gastric procedures for example may be harmed by implementing a broad approach to holding PPI's. While the PPI may be masquerading as an inferior MI, more data is needed to change our clinical practice. In this day of large institutional registries of SPECT myocardial perfusion results, the field of Nuclear Cardiology no longer needs to rely on hypothesis generating studies of one to two hundred patients to determine clinical practice, but instead can marshal adequately powered multicenter investigations to properly address pertinent clinical questions. This is the proper next step for unraveling the story of PPI's and stomach wall uptake.

\section{Disclosure}

Sean R. McMahon and W. Lane Duvall have no conflicts of interest related to this work.

\section{References}

1. Singh H, Mittal B, Sood A, et al. Association of use of proton pump inhibitors and $\mathrm{H} 2$ antagonists with stomach wall uptake in $99 \mathrm{mTc}$ methoxy-isobutyl-isonitrile (MIBI) myocardial perfusion imaging. JNC 2019. https://doi.org/10.1007/s12350-019-01733-9.

2. Gholamrezanezhad A, Moinian D, Eftekhari M, et al. The prevalence and significance of increased gastric wall radiotracer uptake in sestamibi myocardial perfusion SPECT. Int J Cardiovasc Imaging 2006;22:435-41.

3. Goel S, Bommireddipalli S, DePuey EG. Effect of proton pump inhibitors and $\mathrm{H} 2$ antagonists on the stomach wall in $99 \mathrm{mTc}$ sestamibi cardiac imaging. J Nucl Med Technol 2009;37:240-3.

4. Mouden M, Rijkee KS, Schreuder N, et al. Influence of protonpump inhibitors on stomach wall uptake of $99 \mathrm{mTc}$-tetrofosmin in cadmium-zinc-telluride SPECT myocardial perfusion imaging. Nucl Med Commun 2015;36:143-7.

Publisher's Note Springer Nature remains neutral with regard to jurisdictional claims in published maps and institutional affiliations. 